STRUCTURAL BIOLOGY COMMUNICATIONS

ISSN 2053-230X

Received 11 December 2018

Accepted 7 May 2019

Edited by M. W. Bowler, European Molecular Biology Laboratory, France

₹ Current affiliation: Kilimanjaro Christian Medical Centre, Joint Malaria Program, Moshi, Tanzania.

Keywords: peroxiredoxins; archaea; Sulfolobus islandicus; Prx6; chaperones.

PDB reference: 1-Cys peroxiredoxin, 6q5v

Supporting information: this article has supporting information at journals.iucr.org/f

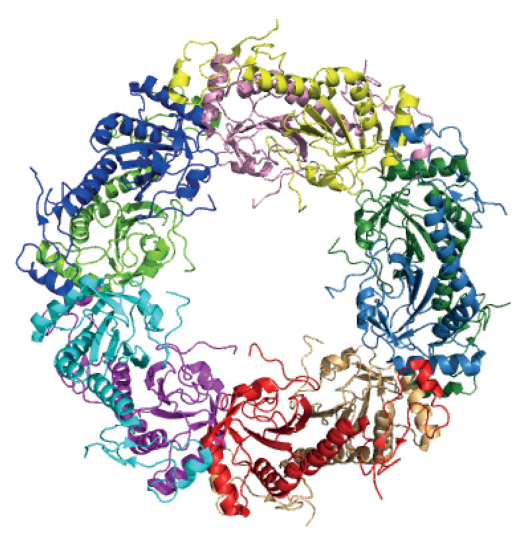

OPEN $\odot$ ACCESS

\section{Structure of the Prx6-subfamily 1-Cys peroxiredoxin from Sulfolobus islandicus}

\author{
Sander Stroobants, ${ }^{a}$ Inge Van Molle, ${ }^{\mathrm{a}, \mathrm{b}}$ Queen Saidi, ${ }^{\mathrm{c}}$ ¥ Karl Jonckheere, \\ Dominique Maes $^{\mathrm{a} *}$ and Eveline Peeters ${ }^{\mathrm{C} *}$
}

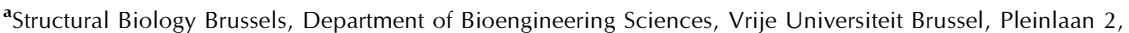
B-1050 Brussels, Belgium, ${ }^{\mathbf{b}}$ VIB-VUB Center for Structural Biology, Pleinlaan 2, B-1050 Brussels, Belgium, and ${ }^{\mathbf{c}}$ Research Group of Microbiology, Department of Bioengineering Sciences, Vrije Universiteit Brussel, Pleinlaan 2, B-1050 Brussels, Belgium. *Correspondence e-mail: dominique.maes@vub.be, eveline.peeters@vub.be
\end{abstract}

Aerobic thermoacidophilic archaea belonging to the genus Sulfolobus harbor peroxiredoxins, thiol-dependent peroxidases that assist in protecting the cells from oxidative damage. Here, the crystal structure of the 1-Cys peroxiredoxin from Sulfolobus islandicus, named 1-Cys SiPrx, is presented. A $2.75 \AA$ resolution data set was collected from a crystal belonging to space group $P 2_{1} 22_{1}$, with unit-cell parameters $a=86.8, b=159.1, c=189.3 \AA, \alpha=\beta=\gamma=90^{\circ}$. The structure was solved by molecular replacement using the homologous Aeropyrum pernix peroxiredoxin (ApPrx) structure as a search model. In the crystal structure, 1-Cys SiPrx assembles into a ring-shaped decamer composed of five homodimers. This quaternary structure corresponds to the oligomeric state of the protein in solution, as observed by size-exclusion chromatography. 1-Cys SiPrx harbors only a single cysteine, which is the peroxidatic cysteine, and lacks both of the cysteines that are highly conserved in the C-terminal arm domain in other archaeal Prx6-subfamily proteins such as ApPrx and that are involved in the association of dimers into higher-molecular-weight decamers and dodecamers. It is thus concluded that the Sulfolobus Prx6-subfamily protein undergoes decamerization independently of arm-domain cysteines.

\section{Introduction}

All organisms need to cope with the presence of reactive oxygen species (ROS) that threaten cellular integrity by damaging nucleic acids, proteins, lipids and other biomolecules. This is certainly the case for thermoacidophilic archaea belonging to the Sulfolobales that live in volcanic hot springs (Brock et al., 1972), which are strongly oxidative habitats. Furthermore, these organisms have a metabolism that is obligately dependent on aerobic respiration and thus generates an additional excessive amount of toxic ROS as byproducts (She et al., 2001; Chen et al., 2005). Therefore, Sulfolobus species harbor a variety of defensive enzymes and antioxidant molecules that are responsible for the detoxification of ROS. Despite the absence of catalase enzymes, antioxidant enzymes named peroxiredoxins (Prxs), which are ubiquitously present in all three domains of life (Schröder \& Ponting, 1998; Wood et al., 2003), constitute an important element of the ROS defense system in Sulfolobus spp. (Limauro et al., 2006). Prx proteins reduce hydrogen peroxide and organic peroxides to water and the corresponding alcohols, respectively, by making use of a thiol-dependent mechanism involving catalytic cysteine residues (Wood et al., 2003). The diverse family of Prxs has been divided into six subfamilies based on active-site profiling (Soito et al., 2011). Archaeal Prxs belong to one of two subfamilies, BCP/PrxQ or Prx6 (Soito et al., 2011), with the latter subfamily typically 
Table 1

Overview of the genes encoding peroxiredoxins in Sulfolobus spp.

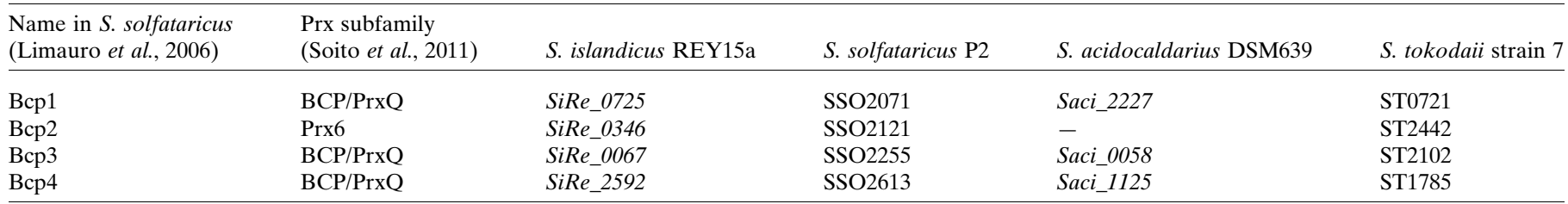

being 1-Cys Prxs that are characterized by a peroxidatic cysteine residue $\left(C_{P}\right)$, which is the sole redox-active cysteine, and thus lacking the resolving cysteine $\left(\mathrm{C}_{\mathrm{R}}\right)$ that is present in 2-Cys Prxs and forms a disulfide bond to the oxidized $C_{P}$.

Sulfolobus species generally harbor four different Prxs, originally named Bcp1, Bcp2, Bcp3 and Bcp4 (Limauro et al., 2006), with Bcp2 belonging to the Prx6 subfamily of 1-Cys Prxs and the others being BCP/PrxQ members (Table 1; Soito et al., 2011). All four Prxs have been experimentally characterized in S. solfataricus (Limauro et al., 2006, 2008, 2010). The 1-Cys Prx Prx6 member is highly conserved in archaea, with $61 \%, 57 \%$ and $55 \%$ sequence identity between S. islandicus Bcp2 (here named 1-Cys SiPrx) and its orthologs from Aeropyrum pernix (ApPrx), Pyrococcus horikoshii (PhPrx) and Thermococcus kodakarensis (TkPrx), respectively (Fig. 1). Furthermore, there is a significant degree of identity to 1-Cys
Prxs from bacteria, humans, Arabidopsis and yeast cells (Lee et al., 2015). In contrast to archaeal 2-Cys Prxs from the BCP/ PrxQ subfamily, such as Bcp1 and Bcp4 from Sulfolobus spp., 1-Cys Prxs such as TkPrx and $S$. solfataricus Bcp2 are not constitutively expressed but instead display an induced expression level in response to oxidative stress (Limauro et al., 2006; Lee et al., 2015). The conserved N-terminal cysteine has been shown to be the peroxidatic $\mathrm{C}_{\mathrm{P}}$ responsible for $\mathrm{H}_{2} \mathrm{O}_{2}$ detoxification (Limauro et al., 2006; Lee et al., 2015; Fig. 1). Besides peroxidase activity, archaeal 1-Cys Prxs are moonlighting enzymes that also have a chaperone activity, thereby protecting proteins and DNA from damage caused by oxidative and thermal stress (Limauro et al., 2006; Lee et al., 2015). For TkPrx, it has been shown that two C-terminal cysteines are responsible for a redox-dependent switch between a dimeric and a higher oligomeric state, with the former mainly
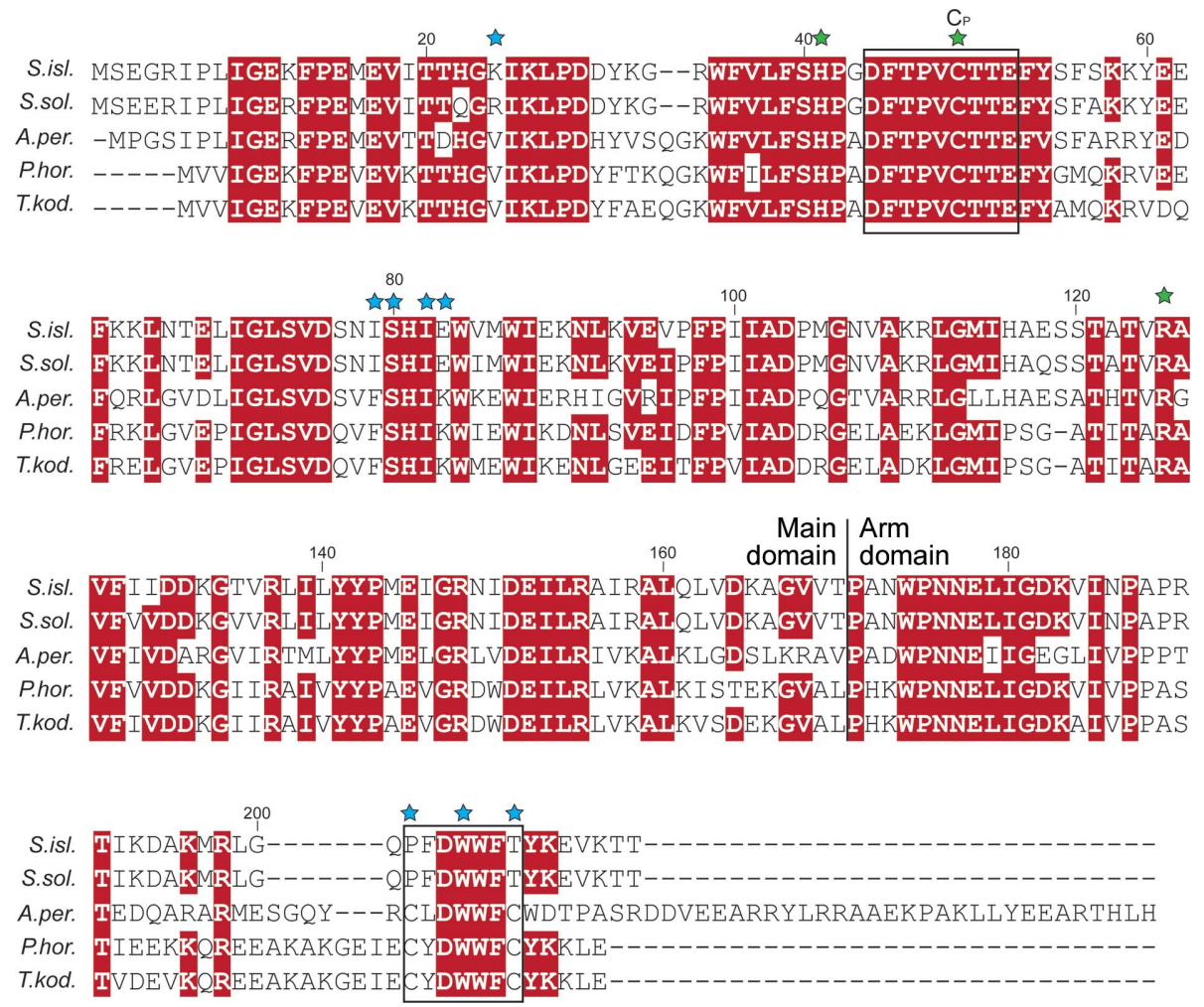

Figure 1

Amino-acid sequence alignment of archaeal Prx6-subfamily 1-Cys peroxiredoxins. S.isl, Sulfolobus islandicus REY15a (ADX84439.1); S.sol., Sulfolobus solfataricus P2 (P95895; 95\% sequence identity); A.per., Aeropyrum pernix K1 (Q9Y9L0.1; 61\% sequence identity); P.hor., Pyrococcus horikoshii OT3 (NP_143112.1; 57\% sequence identity); T.kod., Thermococcus kodakarensis KOD1 (YP_182950.1; 55\% sequence identity). (Highly) conserved residues are indicated in red and conserved motifs are boxed. Residues that are important for peroxidase activity are indicated with a green star (the peroxidatic cysteine is labeled $\mathrm{C}_{\mathrm{P}}$ ), while residues shown to be involved in (redox-sensitive) oligomerization of the protein are indicated with a blue star. Sequence alignment was performed with ClustalW (Larkin et al., 2007) and graphically prepared with Adobe Illustrator. 
Table 2

Macromolecule production.

\begin{tabular}{ll}
\hline Source organism & S. islandicus REY15a \\
DNA source & Genomic DNA \\
Forward primer & 5'-CATGCCATGGTGAGTGAGGGAAGAATT \\
CCATTAATAG-3' & ''-CCGCTCGAGTGTCGTTTTAACTTCTTT \\
Reverse primer & ATAAGTGAAC-3' \\
Cloning vector & pET-28b (Novagen) \\
Expression vector & pET-28b (Novagen) \\
Expression host & E. coli Rosetta \\
Complete amino-acid sequence & MVSEGRIPLIGEKFPEMEVITTHGKIKLPD \\
\multicolumn{1}{c}{ of the construct produced } & DYKGRWFVLFSHPGDFTPVCTTEFYSFS \\
& KKYEEFKKLNTELIGLSVDSNISHIEWV \\
& MWIEKNLKVEVPFPIIADPMGNVAKRLG \\
& MIHAESSTATVRAVFIIDDKGTVRLILY \\
& YPMEIGRNIDEILRAIRALQLVDKAGVV \\
& TPANWPNNELIGDKVINPAPRTIKDAKM \\
& RLGQPFDWWFTYKEVKTTLEHHHHHH \\
\hline
\end{tabular}

functioning as a peroxidase and the latter mainly performing a molecular chaperone function (Lee et al., 2015). Intriguingly, while these C-terminal cysteines are highly conserved in all other archaeal 1-Cys Prxs, the Sulfolobus Prx6-subfamily 1-Cys Prx proteins lack both of them and only have a single cysteine that is the $C_{P}$ (Fig. 1), thus evoking questions about its oligomeric state.

ApPrx and PhPrx have been structurally characterized (Mizohata et al., 2005; Nakamura et al., 2006, 2010, 2013). In contrast, no structural information was available about this Prx enzyme in Sulfolobus spp. Here, we describe the crystallization and structure determination of 1-Cys SiPrx from $S$. islandicus, a highly conserved homolog (95\% sequence identity) of Bcp2 from the closely related $S$. solfataricus.

\section{Materials and methods}

2.1. Heterologous expression and purification of 1-Cys SiPrx

The coding sequence for $S$. islandicus 1-Cys SiPrx (SiRe_0346) was cloned into the NcoI and XhoI restriction sites of pET-28b to generate the pET-28bxSiRe_0346 expression vector (Table 2). Escherichia coli Rosetta cells were transformed with this plasmid vector and cultivated in lysogeny broth (LB) medium supplemented with $60 \mu \mathrm{g} \mathrm{ml}^{-1}$ kanamycin at $310 \mathrm{~K}$. The recombinant expression of the C-terminally His-tagged protein was induced by the addition of $0.1 \mathrm{~m} M$ isopropyl $\beta$-D-1-thiogalactopyranoside (IPTG) at an optical density $\left(\mathrm{OD}_{600 \mathrm{~nm}}\right)$ of 0.7 , followed by further incubation at $310 \mathrm{~K}$ overnight. After centrifugation, the cells were resuspended in buffer $A\left(20 \mathrm{~m} M \mathrm{Na}_{2} \mathrm{HPO}_{4}, 500 \mathrm{~m} M\right.$ $\mathrm{NaCl}, 40 \mathrm{~m} M$ imidazole $\mathrm{pH}$ 7.4) and lysed by sonication for $15 \mathrm{~min}$ at $20 \%$ of the maximal amplitude $(750 \mathrm{~W}$ VibraCell sonicator, Bioblock Sciences). After centrifugation for $10 \mathrm{~min}$ at $15000 \mathrm{~g}$, the supernatant was loaded onto a $5 \mathrm{ml}$ HisTrap FF column (GE Healthcare) equilibrated with buffer $A$ and the protein was eluted using a linear gradient to $500 \mathrm{~m} M$ imidazole in the same buffer using an ÄKTA FPLC system (GE Healthcare). Finally, dialysis was performed against $10 \mathrm{mM}$ Tris- $\mathrm{HCl} \mathrm{pH} 7.5$ or $50 \mathrm{~m} M \mathrm{Na}_{2} \mathrm{HPO}_{4} \mathrm{pH} 7.4,150 \mathrm{~m} M \mathrm{NaCl}$, depending on the downstream analysis.
Table 3

Data-collection and refinement statistics.

Values in parentheses are for the outer shell.

\begin{tabular}{ll}
\hline Data collection & ID29, ESRF \\
X-ray source & PILATUS 6M \\
Detector & 0.976 \\
Wavelength $(\AA)$ & $P 2_{1} 2_{1} 2_{1}$ \\
Space group & $a=86.8, b=159.1, c=189.3$, \\
Unit-cell parameters $\left(\AA{ }^{\circ}{ }^{\circ}\right)$ & $\alpha=90.0, \beta=90.0, \gamma=90.0$ \\
& $76.19-2.75(2.86-2.75)$ \\
Resolution range, spherical $(\AA)$ & $a=3.65, b=2.76, c=2.55$ \\
Resolution range, ellipsoidal $(\AA)$ & $0.095(0.957)$ \\
$R_{\text {merge }}$ & $74.6(33.0)$ \\
Completeness, spherical $(\%)$ & $94.5(93.9)$ \\
Completeness, ellipsoidal $(\%)$ & $285938(14834)$ \\
Total reflections & $51577(2580)$ \\
Unique reflections & $5.5(5.7)$ \\
Multiplicity & $12.7(1.9)$ \\
$\langle I / \sigma(I)\rangle$ & 66.2 \\
Wilson $B$ factor $\left(\AA^{2}\right)$ & $0.999(0.776)$ \\
CC & \\
Refinement & $76.2-2.75$ \\
Resolution range $(\AA)$ & $51522(2221)$ \\
No. of reflections & $21.3(38.6) / 22.8(40.6)$ \\
$R_{\text {cryst }} / R_{\text {free }}(\%)$ & 16820 \\
Protein atoms & 0 \\
Water molecules & 0.102 \\
R.m.s.d., bond lengths $(\AA)$ & 1.60 \\
R.m.s.d., bond angles $\left({ }^{\circ}\right)$ & 62.0 \\
Average $B$ factor $\left(\AA^{2}\right)$ & \\
Ramachandran plot, residues in $(\%)$ & 93.2 \\
Favored region & 6.8 \\
Allowed region & \\
\hline
\end{tabular}

\subsection{Crystallization}

Screening for crystallization conditions was performed by sitting-drop vapor diffusion in two 96-well plates using the Index HT (Hampton Research) and JCSG-plus (Molecular Dimensions) screens at $293 \mathrm{~K}$. Various conditions led to crystallization, with most containing $\mathrm{MgCl}_{2} \cdot 6 \mathrm{H}_{2} \mathrm{O}$ as the precipitant and PEG as a crowding agent. Optimization of the crystallization conditions of 1-Cys SiPrx was performed by the hanging-drop vapor-diffusion method, in which $2 \mu$ l protein solution (7.5 mg ml $\mathrm{mg}^{-1}$ in $10 \mathrm{~m} M$ Tris- $\mathrm{HCl} \mathrm{pH} \mathrm{7.5)} \mathrm{was} \mathrm{mixed}$ with $2 \mu \mathrm{l}$ reservoir solution and equilibrated against $200 \mu \mathrm{l}$ of the same reservoir solution, followed by incubation at $283 \mathrm{~K}$. The optimal reservoir solution was found to be $10 \mathrm{~m} M$ Tris$\mathrm{HCl} \mathrm{pH} 7.5,15 \%(w / v)$ PEG 1500, $0.1 M \mathrm{MgCl}_{2}$. Using this condition, diffracting crystals were obtained after a two-week incubation.

\subsection{Data collection, processing and structure determination}

1-Cys SiPrx crystals were submerged in the original reservoir solution with $35 \%(w / v)$ PEG 1500 for cryoprotection and flash-cooled in liquid nitrogen at $100 \mathrm{~K}$. Diffraction data were collected on beamline ID29 at the ESRF, Grenoble, France using a PILATUS 6M detector. Data sets were obtained from four different crystals, but only data from a single crystal were used for structure determination. The first 1500 images of the collected data were processed using AutoPROC (Vonrhein et al., 2011). As the diffraction data were found to be anisotropic, STARANISO (Tickle et al., 2018) was used to process and scale the final data. The elliptical resolution cutoffs for the $a^{*}$, 
$b^{*}$ and $c^{*}$ axes were determined to be $3.65,2.76$ and $2.55 \AA$, respectively. Based on this information, a resolution cutoff of $2.75 \AA$ was selected. Consequently, the spherical completeness is low both overall and in the highest resolution shell. However, the completeness exceeds $95 \%$ in all shells up to a resolution of $3.4 \AA$.

Molecular replacement was performed with Phaser (McCoy et al., 2007), as included in the PHENIX suite (Adams et al., 2010), using the ApPrx structure from A. pernix (PDB entry $3 \mathrm{a} 2 \mathrm{v}$; Nakamura et al., 2010) with $61 \%$ sequence identity as a model. An ApPrx monomer was used as the search model and the first round of the search resulted in an asymmetric unit

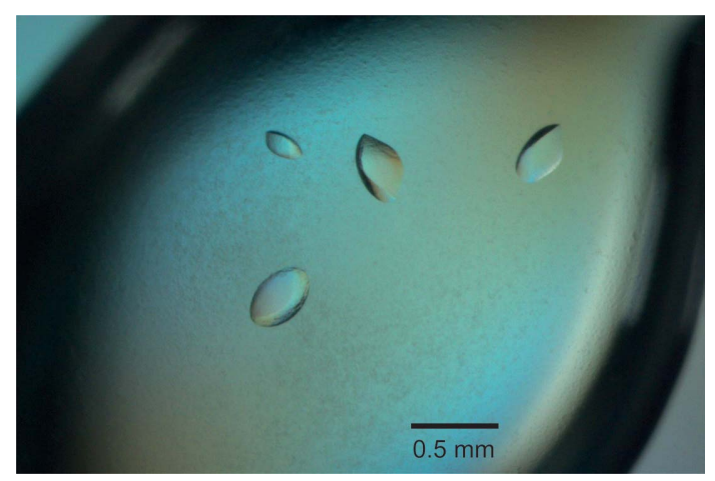

Figure 2

Crystals of S. islandicus 1-Cys SiPrx.

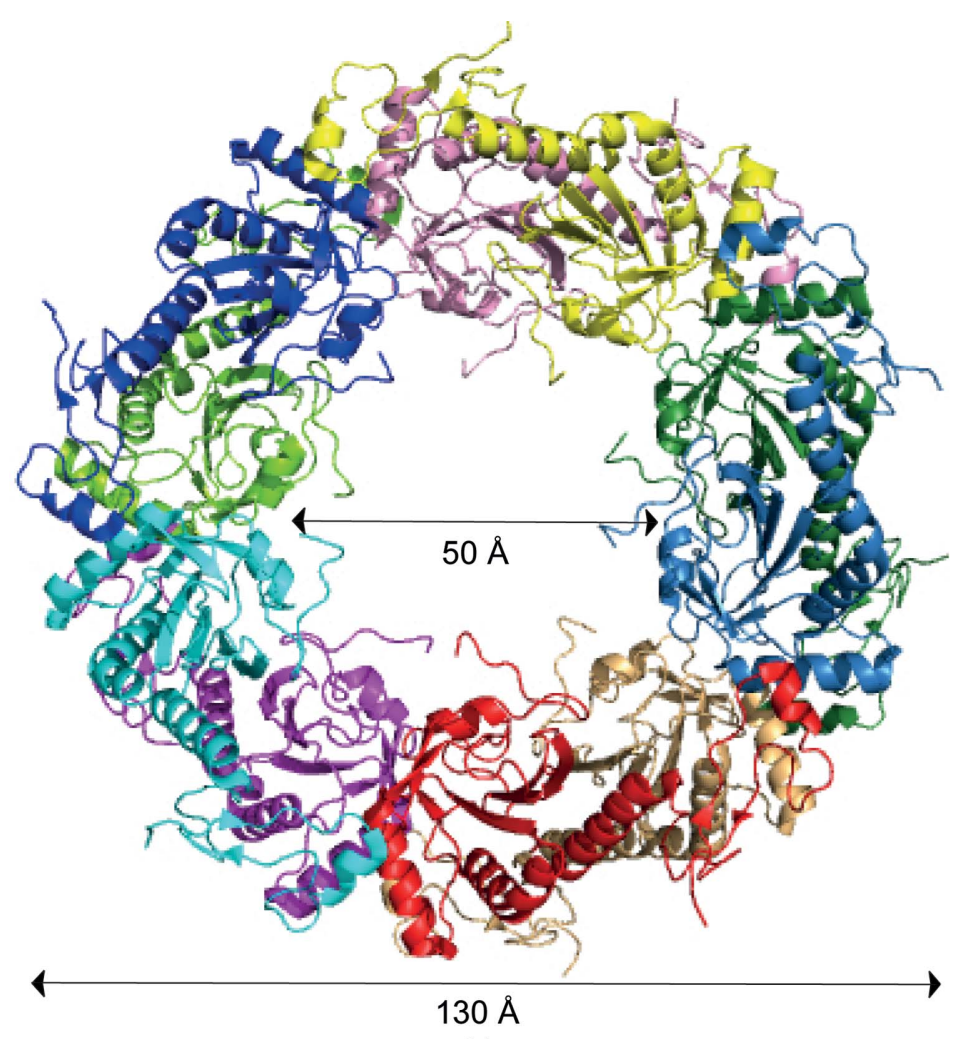

(a) with ten monomer copies. The initial SiPrx model was created using AutoBuild (Adams et al., 2010) and was further built manually using Coot (Emsley et al., 2010). Refinement was performed with PHENIX (Adams et al., 2010) using noncrystallographic constraints and TLS refinement. The quality of the structure was analyzed with MolProbity (Chen et al., 2010). Given the lack of electron density at the N-terminal and C-terminal ends, the first four and the last two residues were not modeled, including the His tag. The data-collection and refinement statistics are summarized in Table 3. The crystal structure has been deposited in the Protein Data Bank (PDB) with accession code $6 \mathrm{q} 5 \mathrm{v}$. All figures displaying protein structures were prepared with $P y M O L$ (DeLano, 2002) and UCSF Chimera 1.12 (Pettersen et al., 2004).

\subsection{Size-exclusion chromatography}

For size-exclusion chromatography (SEC), about $2 \mathrm{mg}$ of 1-Cys Prx protein was mixed with $0.5 \mathrm{mg}$ blue dextran and migrated on a Superdex 200 column (GE Healthcare) using an ÄKTA FPLC system (GE Healthcare) with $50 \mathrm{mM} \mathrm{Na}_{2} \mathrm{HPO}_{4}$ $\mathrm{pH} 7.4,150 \mathrm{mM} \mathrm{NaCl}$ as the running buffer. The following proteins were used to obtain the molecular-weight standard curve: thyroglobulin $(670 \mathrm{kDa}), \gamma$-globulin $(158 \mathrm{kDa})$, ovalbumin $(44 \mathrm{kDa})$ and myoglobulin $(17 \mathrm{kDa})$.

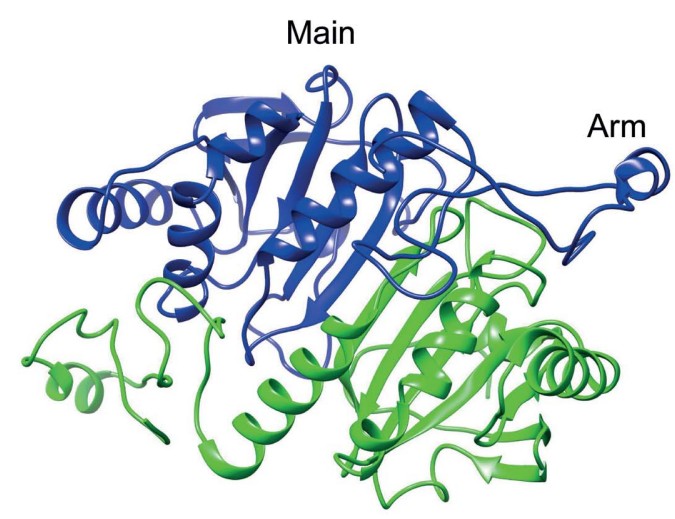

(b)

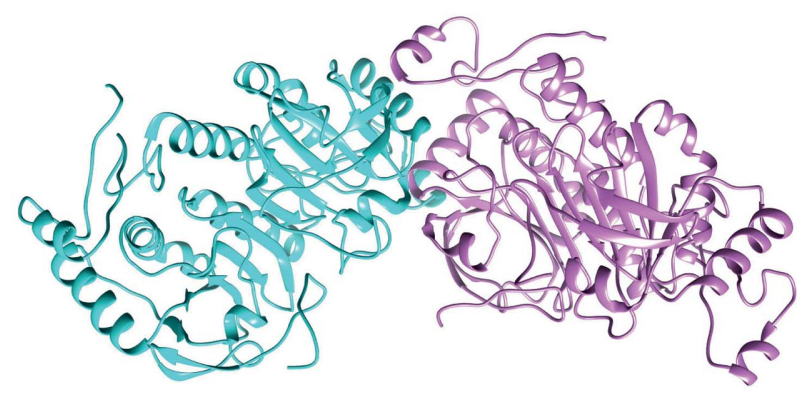

(c)

Figure 3

Structure of 1-Cys SiPrx. (a) Decameric structure, with each monomer colored differently and with the average dimensions indicated. (b) Dimer structure, with each monomer colored differently and with the main and arm-domain regions indicated. (c) Dimer-dimer interface, with each dimer colored differently to emphasize the dimer-dimer interactions. 


\section{Results and discussion}

\subsection{Structure of 1-Cys SiPrx}

Crystals of 1-Cys SiPrx appeared after 30 days of incubation using the hanging-drop vapor-diffusion method (Fig. 2). The crystal structure of 1-Cys SiPrx was determined at $2.75 \AA$ resolution, with $R_{\text {cryst }}$ and $R_{\text {free }}$ values of 21.3 and $22.8 \%$, respectively (Table 3; Fig. 3). The asymmetric unit contained ten subunits arranged in a symmetrical toroid-shaped ring of five dimers (Fig. 3a). Two domains can be discerned in the monomeric structure: an N-terminal main domain and a smaller C-terminal arm domain (Fig. 3b). The protein dimerizes with an extensive monomer-monomer interface created between the main domains involving the formation of a central antiparallel $\beta$-sheet, whereas the two arm domains stick out of the dimeric structure (Fig. $3 b$ ). In this structure, $21 \%$ of the total accessible surface area of a monomer is buried upon dimerization. Dimer-dimer interactions consist of contacts both between main domains and between adjacent main and arm domains (Fig. 3c).

The quaternary structure of 1-Cys SiPrx is similar to those of PhPrx and ApPrx (Mizohata et al., 2005; Nakamura et al., 2006, 2013; Lee et al., 2015). The dimensions of the decameric

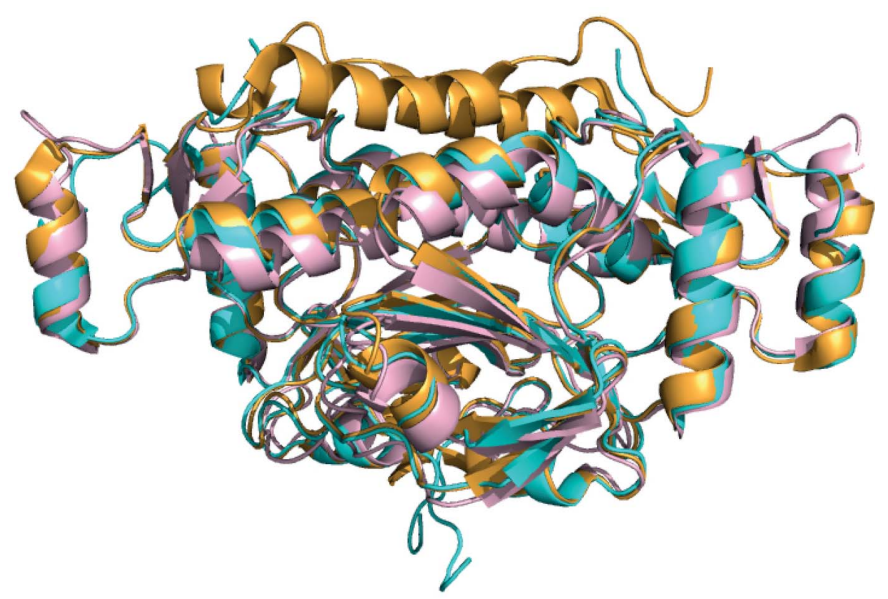

(a)

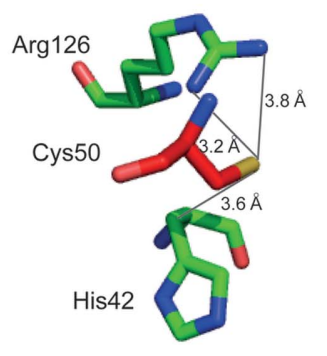

ApPrx

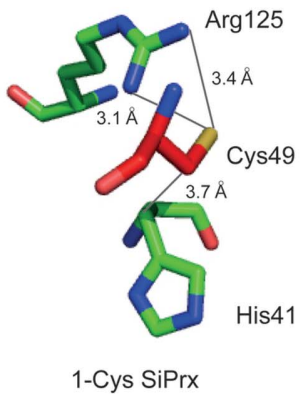

(b)
Figure 4

Structural alignment of 1-Cys SiPrx with other archaeal Prxs. (a) Superimposition of 1-Cys SiPrx (blue; PDB entry 6q5v), ApPrx (orange; PDB entry 3a2v) and PhPrx (pink; PDB entry $3 w 6 g$ ) with the N-terminal ends oriented downwards and the C-terminal ends oriented upwards. (b) Enlarged image displaying the conformations of the active-site residues in ApPrx and 1-Cys SiPrx. The peroxidatic $\mathrm{C}_{\mathrm{P}}$, which is oriented in front of the other two residues, is colored red and the distances to its $\mathrm{S}$ atom are indicated. ring agree well with those observed for these homologous archaeal Prxs, which also form decameric rings (Fig. 3a). A superimposition demonstrates the similarity of the monomeric 1-Cys SiPrx, PhPrx and ApPrx structures, with the exception of the C-terminal tail that is unique to ApPrx and is absent in 1-Cys SiPrx (Fig. 4a). The active site, formed by the peroxidatic Cys49, His41 and Arg125, has the same conformation as those of ApPrx and PhPrx (Fig. 4b). In contrast to the ApPrx structure, in which citrate was present in the active site (Nakamura et al., 2013), the $\mathrm{H}_{2} \mathrm{O}_{2}$-binding pocket of 1-Cys SiPrx was found to be empty.

\subsection{Oligomeric state of 1 -Cys SiPrx in solution}

SEC analysis demonstrated that in solution 1-Cys SiPrx exists as a heterogeneous population of a major $242 \mathrm{kDa}$ species (peak 1) and a minor $506 \mathrm{kDa}$ species (peak 2) (Figs. $5 a$ and $5 b$ ). Taking into account the monomeric molecular weight of $24.7 \mathrm{kDa}$, we conclude that 1-Cys SiPrx mainly exists in a decameric oligomeric form corresponding to the quaternary structure observed in the crystal structure, and that a smaller population exists of a higher-order oligomer, which is probably a 20-subunit protein. ApPrx, PhPrx and TkPrx also form decameric oligomeric states in solution in untreated (nonreducing) conditions (Nakamura et al., 2006, 2013; Lee et al., 2015). TkPrx forms dimers in reducing conditions, while for both TkPrx and PhPrx it has been shown that overoxidation leads to dodecameric structures (Lee et al., 2015; Nakamura et al., 2017).

Archaeal 1-Cys Prxs are characterized by a CXDWWFC motif in the C-terminal arm domain (Mizohata et al., 2005; Lee et al., 2015; Nakamura et al., 2017). Both cysteines are highly conserved in the archaeal enzymes and contribute to the redox-dependent association of dimers into higher-order oligomers (Lee et al., 2015; Nakamura et al., 2017). This is exemplified by mutational analysis of TkPrx: upon mutating one of the two cysteines to a serine, the oligomeric state of the protein becomes dimeric instead of decameric (Lee et al., 2015). Furthermore, upon overoxidation by $\mathrm{H}_{2} \mathrm{O}_{2}$ treatment, leading to the dodecameric form, an intramolecular disulfide bridge is established between these cysteines in PhPrx (Nakamura et al., 2017). It could be hypothesized that disulfidebridge formation converts oxidative stress signals into subtle conformational changes that affect the oligomeric state. Exceptionally, Sulfolobus 1-Cys Prx lacks both cysteines but nevertheless forms a decamer, suggesting that it has lost the redox-sensing function and that it is capable of forming higher oligomeric states independently of the arm-domain cysteines.

Besides the two cysteine residues, all other residues that contribute to transitions between dimers and higher oligomeric states (Nakamura et al., 2017) are conserved or have similar properties, with the exception of the residue at the position corresponding to Lys24: ApPrx and PhPrx harbor a valine at the homologous position.

In conclusion, although untreated 1-Cys SiPrx behaves mainly as a decamer, like other archaeal Prx6-subfamily proteins, it could be postulated that the protein is less sensitive 


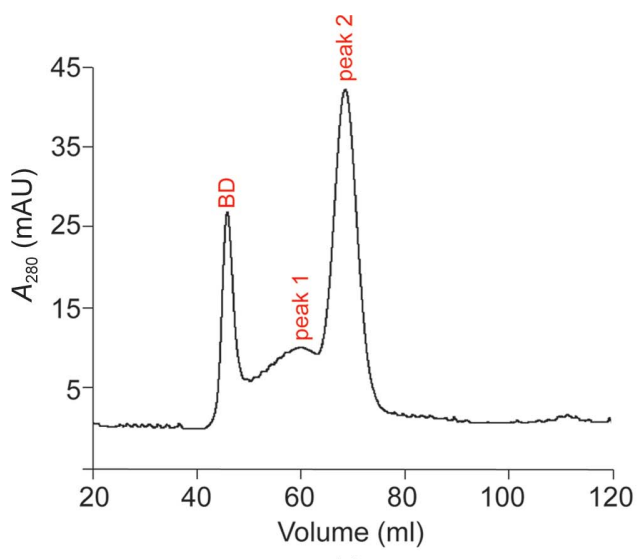

(a)

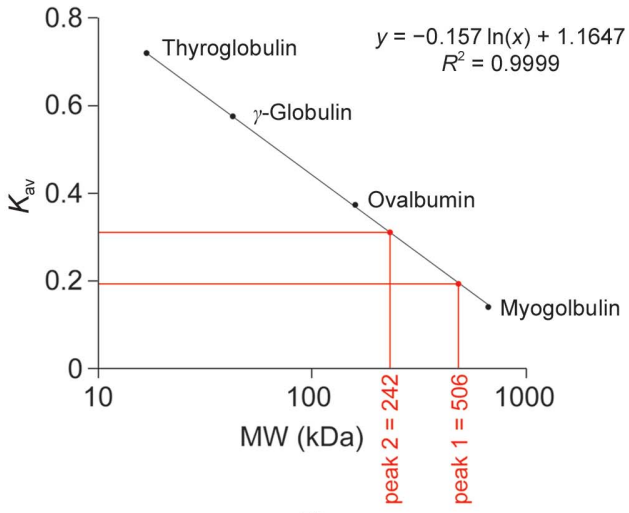

(b)

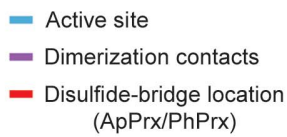

Figure 5

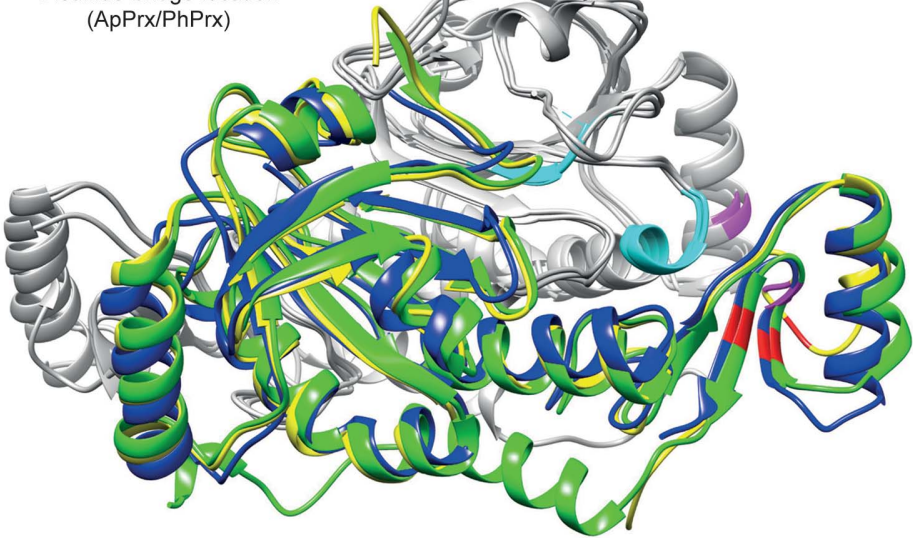

(c)

Oligomeric state of 1-Cys SiPrx in solution and the residues involved in oligomerization. (a) SEC chromatogram indicating the peak corresponding to blue dextran (BD), which was used for determination of the void volume $V_{0}$, and 1-Cys SiPrx peaks 1 and 2. (b) Calibration curve used for the calculation of the molecular weights of the 1-Cys SiPrx peaks. (c) Superimposition of dimeric portions of the 1-Cys SiPrx, ApPrx and PhPrx structures. For each dimer, one monomer is depicted in gray and the other in yellow (SiPrx), blue (ApPrx) or green (PhPrx). Residues that are involved in crucial functions of the protein are colored as indicated.

to redox signaling for oligomeric state conversions and that the decamer is the dominant form. Since it has been shown for TkPrx that higher nondimeric oligomeric forms function not only as peroxidases but mostly as chaperones (Lee et al., 2015), it could be hypothesized that the chaperone function of 1-Cys SiPrx is more specialized compared with that of TkPrx.

\section{Acknowledgements}

Professor Remy Loris helped with affirming the data analysis.

\section{Funding information}

Funding was provided by BelSPO (ESA-Prodex AO-2004070) to SS and DM. Research in the laboratory of EP was supported by the Research Council of the Vrije Universiteit Brussel (start-up funds) and by FWO-Vlaanderen (research project G021118 and research grant $1526418 \mathrm{~N}$ ).

\section{References}

Adams, P. D., Afonine, P. V., Bunkóczi, G., Chen, V. B., Davis, I. W., Echols, N., Headd, J. J., Hung, L.-W., Kapral, G. J., Grosse-
Kunstleve, R. W., McCoy, A. J., Moriarty, N. W., Oeffner, R., Read, R. J., Richardson, D. C., Richardson, J. S., Terwilliger, T. C. \& Zwart, P. H. (2010). Acta Cryst. D66, 213-221.

Brock, T. D., Brock, K. M., Belly, R. T. \& Weiss, R. L. (1972). Arch. Mikrobiol. 84, 54-68.

Chen, L., Brügger, K., Skovgaard, M., Redder, P., She, Q., Torarinsson, E., Greve, B., Awayez, M., Zibat, A., Klenk, H.-P. \& Garrett, R. A. (2005). J. Bacteriol. 187, 4992-4999.

Chen, V. B., Arendall, W. B., Headd, J. J., Keedy, D. A., Immormino, R. M., Kapral, G. J., Murray, L. W., Richardson, J. S. \& Richardson, D. C. (2010). Acta Cryst. D66, 12-21.

DeLano, W. L. (2002). PyMOL. http://www.pymol.org.

Emsley, P., Lohkamp, B., Scott, W. G. \& Cowtan, K. (2010). Acta Cryst. D66, 486-501.

Larkin, M. A., Blackshields, G., Brown, N. P., Chenna, R., McGettigan, P. A., McWilliam, H., Valentin, F., Wallace, I. M., Wilm, A., Lopez, R., Thompson, J. D., Gibson, T. J. \& Higgins, D. G. (2007). Bioinformatics, 23, 2947-2948.

Lee, S., Jia, B., Liu, J., Pham, B. P., Kwak, J. M., Xuan, Y. H. \& Cheong, G.-W. (2015). PLoS One, 10, e0125325-16.

Limauro, D., D'Ambrosio, K., Langella, E., De Simone, G., Galdi, I., Pedone, C., Pedone, E. \& Bartolucci, S. (2010). Biochimie, 92, 14351444.

Limauro, D., Pedone, E., Galdi, I. \& Bartolucci, S. (2008). FEBS J. 275, 2067-2077. 
Limauro, D., Pedone, E., Pirone, L. \& Bartolucci, S. (2006). FEBS J. 273, 721-731.

McCoy, A. J., Grosse-Kunstleve, R. W., Adams, P. D., Winn, M. D., Storoni, L. C. \& Read, R. J. (2007). J. Appl. Cryst. 40, 658-674.

Mizohata, E., Sakai, H., Fusatomi, E., Terada, T., Murayama, K., Shirouzu, M. \& Yokoyama, S. (2005). J. Mol. Biol. 354, 317-329.

Nakamura, T., Kado, Y., Yamaguchi, T., Matsumura, H., Ishikawa, K. \& Inoue, T. (2010). J. Biochem. 147, 109-115.

Nakamura, T., Mori, A., Niiyama, M., Matsumura, H., Tokuyama, C., Morita, J., Uegaki, K. \& Inoue, T. (2013). Acta Cryst. F69, 719-722.

Nakamura, T., Oshima, M., Yasuda, M., Shimamura, A., Morita, J. \& Uegaki, K. (2017). J. Biochem. 162, 415-422.

Nakamura, T., Yamamoto, T., Inoue, T., Matsumura, H., Kobayashi, A., Hagihara, Y., Uegaki, K., Ataka, M., Kai, Y. \& Ishikawa, K. (2006). Proteins, 62, 822-826.

Pettersen, E. F., Goddard, T. D., Huang, C. C., Couch, G. S., Greenblatt, D. M., Meng, E. C. \& Ferrin, T. E. (2004). J. Comput. Chem. 25, 1605-1612.
Schröder, E. \& Ponting, C. P. (1998). Protein Sci. 7, 2465-2468.

She, Q., Singh, R. K., Confalonieri, F., Zivanovic, Y., Allard, G., Awayez, M. J., Chan-Weiher, C. C.-Y., Clausen, I. G., Curtis, B. A., De Moors, A., Erauso, G., Fletcher, C., Gordon, P. M. K., Heikampde Jong, I., Jeffries, A. C., Kozera, C. J., Medina, N., Peng, X., ThiNgoc, H. P., Redder, P., Schenk, M. E., Theriault, C., Tolstrup, N., Charlebois, R. L., Doolittle, W. F., Duguet, M., Gaasterland, T., Garrett, R. A., Ragan, M. A., Sensen, C. W. \& Van der Oost, J. (2001). Proc. Natl Acad. Sci. USA, 98, 7835-7840.

Soito, L., Williamson, C., Knutson, S. T., Fetrow, J. S., Poole, L. B. \& Nelson, K. J. (2011). Nucleic Acids Res. 39, D332-D337.

Tickle, I. J., Flensburg, C., Keller, P., Paciorek, W., Sharff, A., Vonrhein, C. \& Bricogne, G. (2018). STARANISO. Cambridge: Global Phasing Ltd.

Vonrhein, C., Flensburg, C., Keller, P., Sharff, A., Smart, O., Paciorek, W., Womack, T. \& Bricogne, G. (2011). Acta Cryst. D67, 293-302.

Wood, Z. A., Schröder, E., Harris, J. R. \& Poole, L. B. (2003). Trends Biochem. Sci. 28, 32-40. 